\title{
Zeitschriften-Übersicht - Journal Review - Revue des
}

\section{Revues}

Gastroenterology, Vol. 39, December 1960

Postnecrotíc Cirrhosis in Alcoholics. Popper, H., Rubin, E., Krus, S. and Schaffner, F.: Dept. Pathol., Mt. Sinai Hosp., New York, p. 669-686.

Arrested Chronic Ulcerative Colitis. Kiefer, E. D. and Gialanella, R. R. :Lahey Clinic, Boston, Mass., p. 687-689.

Mortality from Ulcerative Colitis in Denmark.

Mosbech, J.: National Health Service, Copenhagen, Denmark, p. 690-693.

Prognosis in Acute and Chronic Regional Enteritis. Gump, F. and Lepore, M. J.: Dept. Surg., Presbyterian Hosp., New York, p. 694-701.

The Effect of Analgesics on Serum Enzymatic Activity. Berk, J. E., Harris, H. and Pringle, B.: Dept. Med., Vet. Admin. Hosp., Dearborn, Mich., p. 702-707.

Diverticula of the Colon and their Relation to the Muscle Layers and Blood Vessels. Slack, W. W.: Dept. Pathol., St. Mark's Hosp., London, England, p. 708-712.

Studies of Hepatic Function with Indocyanine Green. Hunton, D. B., Bollman, J. L. and Hoffman, H. N.: Sect. Biochem., Mayo Clinic, Rochester, Minn., p. 713-724.

Long Term Clinical Studies with R 1132, a New Constipating Drug. Van Derstappen, G., Vantrappen, G. and Vandenbroucke, J.: Dept. Int. Med., Louvain, Belgium, p. 725-729.

Further Observations on Hepatitis and Cirrhosis in Young Women with Positive Clot Tests for Lupus Erythematosus. Bartholomew, L. G., Cain,

J. C, Baggenstoss, A. H. and Hagedom, A. B.: Sect. Med., Mayo Clinic, Rochester, [Minn., p. 730-736.

The Experimental Production of Adenocarcinoma of the Stomach in Rats. Skoryna, S. C. and Ritchie, A. C.: Gastro-Intest. Res. Lab., McGill Univ., Montreal, Canada, p. 737-746.

Injection Studies on the Mesenteric Arterial Circulation. Reiner, L., Platt, R., Rodriguez, F. L. and Jimenez, F. A.: Dept. Pathol., Harvard Med. School, Boston, Mass., p. 747-757.

Demonstration of the Effect of Oral Fat without Choline within One Hour on Centrolobular Hepatic Cells of the Rat. Porta, E. A. and Hart-roft, W. S.: Dept. Pathol. Washington Univ., St. Louis, Miss., p. 758-763.

Mast Cells and the Gastrointestinal Tract. Kraft, S. C. and Kirsner, J. B.: Dept. Med., Univ. Chicago, 111., p. 764-770.

Gastric Secretory Responses to Repeated Intravenous Infusions of Histamine and Gastrin in Nonanesthetized and Anesthetized Gastric Fistula Cats. Etnas, S.: Dept. Pharmacol. Karolinska Inst., Stockholm, Sweden, p. 771-782.

Prolonged Pyrexia from Penetrating Benign Ulcer on the Greater Curvature. Kasich, A. M. and Miller, J. W.: Med. Service, Lenox Hill Hosp., New York, p. 783-786. 
Squamous Cell Carcinoma of the Stomach. Cruze, K., Maas, H. E., Clarke, J. S. and El Farra, S.: Surg. Serv., Vet. Admin. Hosp., Los Angeles, Calif., p. 787-789.

ad 1 . On a $\Gamma$ habitude de décrire la cirrhose alcoolique comme une cirrhose portale. Cependant, il n'est pas rare de trouver les caractères d'une cirrhose post-nécrotique chez des sujets alcooliques. En effets, sur 187 alcooliques, les auteurs trouvent 85 cas de cirrhose post-nécrotiques, soit $45 \%$, alors que sur 73 patients non alcooliques, la proportion de cirrhoses post-nécrotiques est de 79 $\%$. La dégénérescence graisseuse est rare chez les non alcooliques tandis qu'on trouve les corps hyalins de Mallory chez 56,6\% des alcooliques et 2,7\% des sujets sobres. La forme postnécrotique des alcooliques s'accompagne d'un foie moins gros et d'une rate plus impor-tante que dans la cirrhose portale. Les nodules multilobulaires constituent la carac-téristique la plus courante de la cirrhose post-nécrotique. Les stades de transition entre les 2 types de cirrhose sont frequents. II est possible que la cirrhose portale, avec dégénérescence graisseuse, necrose, collapsus puis regeneration, se termine en cirrhose post-nécrotique. La survie prolongée des malades grace à un traitement amélioré est sans doute la cause de $\Gamma$ augmentation de frequence des cirrhoses post-nécrotiques.

ad 3. Au Danemark, de 1951 à 1959, la côlite ulcéreuse a provoqué une mortalité annuelle de 5 personnes pour 1 million d'habitants; le rapport est de 3 hommes pour 4 femmes. Environ la moitié des cas mortels avaient duré 1 an ou moins. La perforation représente la complication la plus fréquemment responsable de la mort (au moins $10 \%$ des cas).

ad 4. 165 cas d'entérite régionale de Crohn ont été observes de 1932 à 1957 au Presbyterian Hospital de New York; 117 de ces malades ont été opérés et suivis pendant une dizaine d'années. Le plus souvent, il s'agissait d'une iléocolectomie et d'une colostomie iléo-transverse, dont la mortalité ne fut que de $1,4 \%$. La frequence des rechûtes varie de $19 \%$ pour les sujets suivis 2 ans à $44 \%$ pour ceux observes 10 ans. La maladie ne parait pas plus bénigne chez les sujets plus âgés. Les cas aigus évo-

Zeitschriften-Übersicht - Journal Review - Revue des Revues 71 luent exceptionnellement en cas chroniques; s'agirait-il de deux entités différentes ? Le groupe de malades «guéris» sans intervention représente le 10,3\%.

ad 9. Description d'un nouveau syndrome survenant chez des femmes jeunes et caractérisé par une affection hépatique sérieuse, une hyperglobulinémie et un test de coagulation positif pour le lupus érythémateux. Parmi les symptômes, on relève une fièvre périodique, une polyarthrite migratrice, une pleurésie, des douleurs abdominales, une sensibilité à la lumière solaire et aux medicaments, des eruptions, de Гasthénie. L'insuffisance hépatique prend ensuite le pas, avec ictère, hépatosplénomégalie et finalement exitus. B. Wissmer (Geneve) Acta gastro-enterologica belgica; Vol. 23, décembre 1960 (Ce numéro fait suite à celui de maijuin 1960)

La chirurgie des pancréatites chroniques* Mallet-Guy, P., Lyon, p. 965-980.

Etude pathogénique et nosologique des pancréatites chroniques de $\Gamma$ adulte. Sarles, H.; Sarles, J. C.; Muratore, R. et Guíen, C.: Hôtel-Dieu, Marseille, p. 981-1001.

3. Mise en evidence d'anticorps antipancréas dans le serum des malades atteints de pancréatite chronique et d'affections pancréatiques diverses.

Schwarzmann, V. et Julien, C.: Hop. Saint-Antoine, Paris, p. 1002-1009. 
A propos de $\Gamma$ utilisation dee hormones duodénales dans le diagnostic des affections panréatiques exocrines. Delcourt, A. et Voordecker, G.: Service gastro-entér. 1'Institut médico-chirurg. d'lxelles, Bruxelles, p. 1010-1015.

Repercussions métaboliques d'une pancréatectomie totale. Tæuf, G. de et Delcourt, A.: Univ. iibre Bruxelles, p. 1016-1028.

Lithiase pancréatique au cours de Thyperthyroï-die. Birnstingl, M.: St. Bartholomew’s Hosp., London 1, p. 1029-1030.

Pancreatic resection for severe chronic pancreatitis. Smith, R.: St. George's Hosp., London, p. 1031-1033.

Indications opératoires et résultats dans 42 cas de pancréatite $\beta$ chroniques diffuses. Razemon, P. et Salembier, Y., Lille, p. 1034-1037.

Note sur les bases de la tactique chirurgicale dans les pancréatites chroniques. Arianoff, A. et Van Dessel, A., Bruxelles, p. 1038-1051.

10. Considerations sur les possibilités du traitement

chirurgical des pancréatites chroniques. Pi-

Figueras, J., Barcelone, p. 1052-1058.

11. Valeur du sommeil périodique provoqué par le

luminal comme un adjuvant dans le traitement

des pancréatites. Bojanowicz, K.; Olszewski,

W. et Narolska, I.: Acad. méd. Lodz (Pologne),

p. 1059-1069.

ad 1. II faut distinguer la lithiase du Wirsung de la microlithiase disséminée péri-phérique et des calcifications parenchymateuses diffuses. La premiere precede la pancréatite et nécessite la désobstruction avec pancréatectomie gauche. A Гinverse, les microcalculs et les calcifications parenchymateuses en aires constituent des «modalités évolutives d'un quelconque processus inflammatoire». Le diagnostic radiologique peropératoire est fait par visualisation descendante $\mathrm{du}$ Wirsung après incision ou ponction du canal, preferable à la voie retrograde. Le facteur patho-génique biliaire est surtout le fait des reflux à la faveur d'une hypotonie oddienne, plus que d'une hypertonie. Dans 32\% des cas, la pancréatite semble être primitive, ne découler ni d'une cause biliaire, ni sphinctérienne, ni wirsungienne. La dilatation canalaire lui est secondaire et ne justifie pas la wirsungo-jejunostomie. La pathogénie des récidives successives est nerveuse, du type phénomène de Reilly. Pancréatectomie gauche si les lesions sont limitées à cette portion de la glande; sinon, splanchni-cectomie gauche et resection du ganglion semi-lunaire.

ad 2. Conception pluraliste de la nosologie des pancréatites, opposée à la conception uniciste américaine. Pancréatites calcifiantes; pancréatites non calcifiantes primitives; pancréatites d'amont, secondaires à un obstacle canalaire (oddite, etc.), non calcifiantes également. Ces pancréatites sont à distinguer des pancréatites aiguës, à poussée unique ou récurrentes, dont les rechutes n'aboutissent pas, en regie géné-rale à la sclérose calcifiante disséminée. Les pancréatites calcifiantes auraient une origine nutritionnelle (excès d'alcool et de graisses, carences protidiques). La distinction entre la lithiase pancréatique et la pancréatite calcifiante parait artificielle. L'étiologie des pancréatites primitives non calcifiantes serait inflammatoire (auto-immunisation ?).

ad 3. La recherche d'anticorps antipancréas dans la pancréatite est positive dans $80 \%$ des cas. Toutefois elle n'est pas spécifique, puisque positive dans $75 \%$ des 